\title{
Comparison of four antibiotics with indigenous marine Bacillus spp. in controlling pathogenic bacteria from shrimp and Artemia
}

\begin{abstract}
Use of antibiotics for the control of bacterial diseases in shrimp culture has caused several adverse impacts to the industry. This has resulted in the search for alternative environment friendly approaches to overcome bacterial infections. This study was conducted to investigate the use of beneficial bacteria as an alternative to antibiotics. Ten pathogenic bacterial species isolated from shrimp, Penaeus monodon, and Artemia cysts were tested for susceptibility to indigenous marine Bacillus subtilis AB65, Bacillus pumilus AB58,Bacillus licheniformis AB69 and compared with oxytetracycline, chloramphenicol, gentamicin and bacitracin, which are common antibiotics used in Asian aquaculture. The Bacillus spp. were isolated from the local marine environment for bioremediation use in shrimp hatcheries and were proven to reduce total ammonium nitrogen. The pathogenic bacterial isolates were $90 \%$ susceptible to B. subtilis AB65, 70\% susceptible to B. pumilus AB58 and B. licheniformis AB69 and 100\% susceptible to oxytetracycline, chloramphenicol and gentamicin but only $40 \%$ to bacitracin. Two representative isolates of the vibrio group, Vibrio alginolyticus VaM11 and Vibrio parahaemolyticus VpM1, when tested for competitive exclusion by a common broth method using the marine Bacillus spp., showed decreased viable counts from 108 to $102 \mathrm{cfu} \mathrm{mL}$ )1.The results suggest that the action of the marine bacteria appears to be significant in protecting the host shrimp against pathogenic bacteria. In addition to the alternative use of antibiotics, the selected marine bacteria had additional bioremediation properties of reducing ammonia.
\end{abstract}

Keyword: alternative to antibiotics, Artemia, Bacillus, inhibition test, marine bacteria, shrimp. 